Global Journal of Pure and Applied Mathematics.

ISSN 0973-1768 Volume 16, Number 6 (2020), pp. 871-889

(C) Research India Publications

https://dx.doi.org/10.37622/GJPAM/16.6.2020.871-889

\title{
A Study on Properties and Goodness-of-Fit of the Logistic Inverse Weibull Distribution
}

\author{
Arun Kumar Chaudhary ${ }^{1}$, Vijay Kumar ${ }^{2}$ \\ ${ }^{1}$ Department of Management Science(Statistics), Nepal Commerce Campus, \\ Tribhuwan University, Kathmandu, Nepal. \\ ${ }^{2}$ Department of Mathematics and Statistics, DDU Gorakhpur University, \\ Gorakhpur-273009, India.
}

\begin{abstract}
In this paper, we purpose a three-parameter univariate continuous distribution called Logistic Inverse Weibull Distribution. We have presented some statistical properties of the purposed distribution including the cumulative distribution function, probability density function, hazard rate function, survival function, quantile function, etc. The three well-known estimation methods namely least-square estimation (LSE), maximum likelihood estimation (MLE), and Cramer-Von-Mises estimation (CVME) methods are used for the model parameter estimation. The overall goodness of fit of the proposed distribution is assessed by comparison with some other existing distributions using a real data set.
\end{abstract}

Keywords: Logistic distribution, Inverse Weibull distribution, survival function, LSE, CVME, MLE

\section{INTRODUCTION}

The Weibull distribution has been extensively used in survival and reliability analysis. For a detailed study, the learners can go through Lai et al. (2003) and Nadarajah (2009). Even though its widespread use, it has some drawbacks that shape of its hazard rate function (HRF) are restricted that can only be monotonically constant or decreasing or increasing. Usually, real-world problems require a broader range of possibilities in the moderate risk, for instance, in the case of biological mortality and 
mechanical lifecycle, when the lifetime data produces a bathtub shaped hazard function.

The Inverse Weibull distribution has been applied to describe many actual life problems. It has been used to model the degradation phenomenon of machine parts like crankshafts of diesel engines, pistons, and failure of insulating fluid. (Khan et al., 2008, Pararai et al., 2014). Akgül et al. (2016) has introduced the inverse Weibull distribution for modeling the wind speed data. Model parameters estimation and reliability properties of Inverse Weibull distribution, based on a random censoring model, and has been presented by Kumar \& Kumar (2019). To obtain greater flexibility in distribution, researchers in the previous years have introduced several modified forms and extensions of Weibull distribution. Some generalizations of the Weibull (W) distribution are found in the statistical literature such as additive W (Xie and Lai, 1996), the exponentiated W (Mudholkar et al., 1996), Marshall-Olkin extended W (Ghitany et al., 2005), beta-W (Lee et al., 2007), beta modified W (Silva et al., 2010), transmuted W (Aryal and Tsokos, 2011), modified W (Lai et al. 2003, Sarhan and Zaindin, 2009), exponentiated generalized W (Cordeiro et al., 2013), Kumaraswamy inverse W (Shahbaz et al., 2012), transmuted complementary W geometric (Afify et al., 2014), beta inverse W (Hanook et al., 2013), Kumaraswamy complementary $\mathrm{W}$ geometric (Afify et al. 2017) distributions and Topp-Leone generated W (Aryal et al. 2017).

Recently, Okasha et al. (2017) has introduced the extended inverse Weibull distribution with reliability application Cordeiro et al. (2018) has introduced the Lindley Weibull distribution which accommodates unimodal and bathtub, and a broad variety of monotone failure rates, Basheer (2019) also introduced the alpha power inverse Weibull distribution with reliability application and Abd EL-Baset \& Ghazal (2020) has presented the exponentiated additive Weibull distribution. Joshi and Kumar (2020) have presented a three-parameter Lindley inverse Weibull distribution having unimodal and bathtub, and a wide variety of monotone failure rates.

The logistic distribution is a univariate continuous distribution that is similar in shape to normal distribution. It is a special case of Tukey lambda distribution. Both its Probability density function and Cumulative distribution functions have been used in many different areas such as logistic regression, modeling growth, neural networks, and logit models. The application of this distribution is seen in areas such as demography, physical sciences, finance, sports modeling, actuarial sciences, etc. In comparison to a normal distribution, the logistic distribution has higher kurtosis (heavier tails) so it provides better insight into the likelihood of extreme events and has more consistency with the underlying data. ( Glantz \& Kissell, 2014) Let $Y$ be a non-negative random variable follows the logistic distribution with shape parameter $\lambda$ $>0$, and its cumulative distribution function is given by

$$
F(x ; \lambda)=\frac{1}{1+e^{-\lambda y}} ; \lambda>0, y \in \mathfrak{R}
$$


and its corresponding PDF is

$$
f(y ; \lambda)=\frac{\lambda e^{-\lambda y}}{\left(1+e^{-\lambda y}\right)^{2}} ; \lambda>0, y \in \mathfrak{R}
$$

Tahir et al. (2016) have presented the logistic-X family which is a new generating family of continuous distributions generated from a logistic random variable. Its probability density function may be reversed-J shaped, symmetrical, right-skewed and, left-skewed and may have a bathtub, upside-down bathtub, decreasing and increasing hazard rates shaped. Lan \& Leemis (2008) has introduced a compounded model called logistic-exponential survival distribution, which consists of Decreasing Failure Rate, Increasing Failure Rate, Upside-Down Bathtub Shaped Failure Rate, and Bathtub Shaped Failure rate. This model would be very useful in lifetime modeling. Unlike most distributions in the Upside-Down Bathtub Failure Rate and Bathtub Shaped Failure Rate classes, the logistic-exponential distribution show closed-form density, survival functions, hazard, and cumulative hazard.

The survival function of the logistic-exponential distribution is

$$
S(x ; \lambda)=\frac{1}{1+\left(e^{\lambda x}-1\right)^{\alpha}} ; \quad \alpha>0, \lambda>0, x \geq 0
$$

Using the same approach used by (Lan \& Leemis, 2008), we purpose a new distribution termed Logistic Inverse Weibull (LIW) distribution. The key objective of this article is to introduce a distribution with higher flexibility by inserting just one more parameter to the inverse Weibull distribution to attain a better fit for the lifetime data sets. We have discussed some statistical properties and their applicability.

The different segments of the proposed study are arranged as follows. In Section 2 we present the Logistic inverse Weibull distribution and its various properties. We have made use of three well-known estimation methods to estimate the model parameters namely the least-square estimation (LSE), maximum likelihood estimation (MLE), and Cramer-Von-Mises estimation (CVME) methods. For the maximum likelihood (ML) estimate, we have constructed the asymptotic confidence intervals using the observed information matrix which are presented in Section 3. In Section 4, a real data set has been analyzed to explore the applications and capability of the proposed distribution.

Here, we calculate the estimated value of the parameters and log-likelihood, Bayesian information criterion (BIC), Akaike information criterion (AIC), Corrected Akaike information criterion (AICC) criterion, and Hannan-Quinn information criterion (HQIC) for ML, LSE, and CVME. Also, the goodness of fit of the proposed distribution is evaluated by fitting it in comparison with some other well-known existing distributions using a real data set. In Section 5 we present the conclusion. 


\section{THE LOGISTIC INVERSE WEIBULL (LIW) DISTRIBUTION}

Following the same method used by (Lan \& Leemis, 2008) we have presented the new distribution called logistic inverse Weibull distribution. Let $X$ be a non-negative random variable with a positive scale parameter $\lambda$ and positive shape parameters $\alpha$ and $\beta$, then $\mathrm{CDF}$ of logistic inverse Weibull distribution can be defined as

$$
F(x)=\frac{1}{1+\left(e^{\lambda x^{-\beta}}-1\right)^{\alpha}} ; \quad(\alpha, \beta, \lambda>0), x>0
$$

And PDF of logistic Inverse Weibull distribution is

$$
f(x)=\frac{\alpha \beta \lambda x^{-(\beta+1)} e^{\lambda x^{-\beta}}\left(e^{\lambda x^{-\beta}}-1\right)^{\alpha-1}}{\left[1+\left(e^{\lambda x^{-\beta}}-1\right)^{\alpha}\right]^{2}} ;(\alpha, \beta, \lambda>0), x>0
$$

This CDF function is comparable to the log-logistic CDF function by changing the second term of the denominator in its base to Inverse Weibull function, hence we named it as logistic Inverse Weibull distribution.

\section{Reliability function /Survival function}

The reliability function/survival function of logistic Inverse Weibull distribution is

$$
R(x)=1-F(x)=1-\frac{1}{1+\left(e^{\lambda x^{-\beta}}-1\right)^{\alpha}} ; \quad(\alpha, \beta, \lambda>0), \quad x>0
$$

\section{Hazard function}

The hazard rate function of logistic Inverse Weibull distribution can be defined as,

$$
h(x)=\frac{f(x)}{R(x)}=\frac{\alpha \beta \lambda x^{-(\beta+1)} e^{\lambda x^{-\beta}}\left(e^{\lambda x^{-\beta}}-1\right)^{\alpha-1}}{\left(e^{\lambda x^{-\beta}}-1\right)^{\alpha}\left[1+\left(e^{\lambda x^{-\beta}}-1\right)^{\alpha}\right]} ;(\alpha, \beta, \lambda>0), x>0
$$

In Figure 1, we have plotted the PDF and failure rate function of logistic Inverse Weibull distribution for various values of $\lambda$ and $\alpha$. 

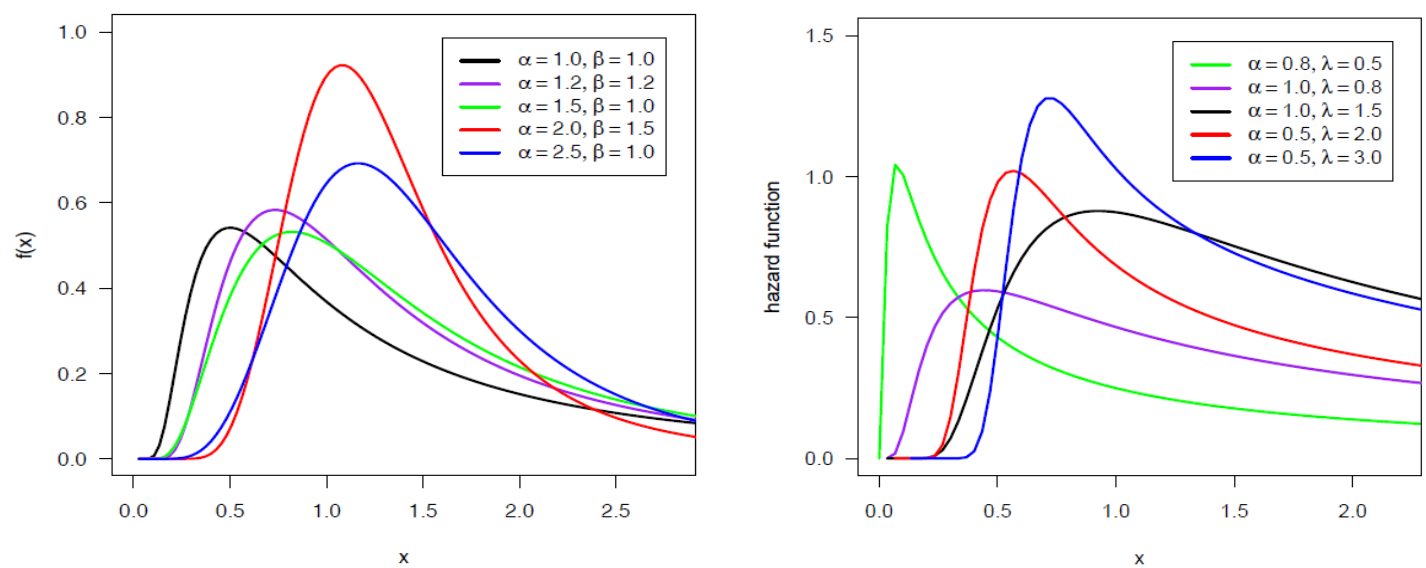

Figure 1. Graphs of PDF (left side) and hazard function (right side) for different values of $\alpha$ and $\lambda$.

\section{Quantile function}

Quantile function of logistic Inverse Weibull distribution can be expressed as

$$
Q(p)=\left[\frac{1}{\lambda} \ln \left\{\left(\frac{1-p}{p}\right)^{1 / \alpha}+1\right\}\right]^{-1 / \beta} ; 0<p<1
$$

\section{Median:}

The median of logistic Inverse Weibull distribution can be expressed as

$$
\text { Median }=\left[\frac{1}{\lambda} \log 2\right]^{-1 / \beta}
$$

Here we notice that the median is dependent upon scale parameter $\beta$ and $\lambda$ only.

Skewness and Kurtosis:

The Bowley's coefficient of skewness based on quartiles is

$$
S_{B}=\frac{Q(0.75)+Q(0.25)-2 Q(0.5)}{Q(0.75)-Q(0.25)} \text { and }
$$

The coefficient of kurtosis based on octiles which were defined by (Moors, 1988) is

$$
K_{\text {MOOR }}=\frac{Q(0.875)-Q(0.625)+Q(0.375)-Q(0.125)}{Q(3 / 4)-Q(1 / 4)}
$$




\section{METHODS OF ESTIMATION}

In this section, the model parameters of the proposed distribution are estimated by applying some well-known estimation methods which are as follows

\subsection{Maximum Likelihood Estimates}

For model parameter estimation, the most commonly used method is the maximum likelihood method (Casella \& Berger, 1990). Let $x_{1}, x_{2}, \ldots x_{n}$ be a random sample generated from $\operatorname{LIW}(\alpha, \beta, \lambda)$. Then, the likelihood function $L(\alpha, \beta, \lambda)$ is given by,

$$
\begin{aligned}
& L\left(\xi ; x_{1}, x_{2} \ldots x_{n}\right)=f\left(x_{1}, x_{2}, \ldots x_{n} / \xi\right)=\prod_{i=1}^{n} f\left(x_{i} / \xi\right) \\
& L(\alpha, \beta, \lambda)=\alpha \beta \lambda \prod_{i=1}^{n} \frac{x^{-(\beta+1)} e^{\lambda x^{-\beta}}\left(e^{\lambda x^{-\beta}}-1\right)^{\alpha-1}}{\left[1+\left(e^{\lambda x^{-\beta}}-1\right)^{\alpha}\right]^{2}} ;(\alpha, \beta, \lambda)>0, x>0
\end{aligned}
$$

Now log-likelihood density is

$$
\ell(\alpha, \beta, \lambda \mid \underline{x})=n \ln (\alpha \beta \lambda)-(\beta+1) \sum_{i=1}^{n} \ln \left(x_{i}\right)+\lambda \sum_{i=1}^{n} x_{i}^{-\beta}+(\alpha-1) \sum_{i=1}^{n} \ln \left\{A\left(x_{i}\right)\right\}-2 \sum_{i=1}^{n} \ln \left\{1+\left[A\left(x_{i}\right)\right]^{\alpha}\right\}
$$

where $A\left(x_{i}\right)=e^{\lambda x^{-\beta}}-1$

Differentiating (3.1.1) with respect to $\alpha, \beta$, and $\lambda$ we get,

$$
\begin{aligned}
& \frac{\partial l}{\partial \alpha}=\frac{n}{\alpha}+\sum_{i=1}^{n} \ln \left\{A\left(x_{i}\right)\right\}-2 \sum_{i=1}^{n} \frac{\left[A\left(x_{i}\right)\right]^{\alpha} \ln \left[A\left(x_{i}\right)\right]}{1+\left[A\left(x_{i}\right)\right]^{\alpha}} \\
& \frac{\partial l}{\partial \beta}=\frac{n}{\beta}-\sum_{i=1}^{n} \ln x_{i}-\beta \lambda \sum_{i=1}^{n} x_{i}^{-(\beta+1)}(\alpha-1) \beta \lambda \sum_{i=1}^{n} \frac{e^{\lambda x_{i}^{-\beta}} x_{i}^{-(\beta+1)}}{A\left(x_{i}\right)}-2 \sum_{i=1}^{n} \alpha \lambda \frac{\ln x_{i} e^{\lambda x_{i}^{-\beta}}}{x_{i}^{\beta}} \frac{\left[A\left(x_{i}\right)\right]^{\alpha-1}}{1+\left[A\left(x_{i}\right)\right]^{\alpha}} \\
& \frac{\partial l}{\partial \lambda}=\frac{n}{\lambda}+\sum_{i=1}^{n} x_{i}^{-\beta}+(\alpha-1) \sum_{i=1}^{n} \frac{x_{i}^{-\beta} e^{\lambda x_{i}^{-\beta}}}{A\left(x_{i}\right)}-2 \alpha \sum_{i=1}^{n} \frac{e^{\lambda x_{i}^{-\beta}}}{x_{i}^{\beta}} \frac{\left[A\left(x_{i}\right)\right]^{\alpha-1}}{1+\left[A\left(x_{i}\right)\right]^{\alpha}}
\end{aligned}
$$

The values of unknown model parameters $\alpha, \beta$, and $\lambda$ can be obtained by equating above three linear equations to zero and solving them simultaneously for $\alpha, \beta$, and $\lambda$. We obtain the corresponding maximum likelihood estimate $\hat{\alpha}, \hat{\beta}$ and $\hat{\lambda}$ of the parameters $\alpha, \beta$, and $\lambda$. To obtain the estimated value of $\alpha, \beta$, and $\lambda$, we have used statistical computing software such as R, Mathematica, Matlab, etc for maximization of equation(3.1) We have to calculate the observed information matrix to construct the confidence interval estimation of $\alpha, \beta$ and $\lambda$ and for testing of the hypothesis. The observed information matrix for $\alpha, \beta$, and $\lambda$ can be obtained as, 
$W=\left[\begin{array}{lll}W_{11} & W_{12} & W_{13} \\ W_{21} & W_{22} & W_{23} \\ W_{31} & W_{32} & W_{33}\end{array}\right]$

Where

$W_{11}=\frac{\partial^{2} l}{\partial \alpha^{2}}, W_{12}=\frac{\partial^{2} l}{\partial \alpha \partial \beta}, W_{13}=\frac{\partial^{2} l}{\partial \alpha \lambda}$

$W_{21}=\frac{\partial^{2} l}{\partial \beta \partial \alpha}, W_{22}=\frac{\partial^{2} l}{\partial \beta^{2}}, W_{23}=\frac{\partial^{2} l}{\partial \beta \partial \lambda}$

$W_{31}=\frac{\partial^{2} l}{\partial \lambda \partial \alpha}, W_{32}=\frac{\partial^{2} l}{\partial \beta \partial \lambda}, W_{33}=\frac{\partial^{2} l}{\partial \lambda^{2}}$

Let $\varpi=(\alpha, \beta, \lambda)$ denote the parameter space and the corresponding MLE of $\varpi \hat{\varpi}=(\hat{\alpha}, \hat{\beta}, \hat{\lambda})$ as, then $(\hat{\varpi}-\varpi) \rightarrow N_{3}\left[0,(W(\varpi))^{-1}\right]$ where $W(\varpi)$ is the Fisher's information matrix. Using the Newton-Raphson algorithm to maximize the likelihood creates the observed information matrix and hence the variance-covariance matrix is obtained as,

$$
[W(\varpi)]^{-1}=\left(\begin{array}{ccc}
\operatorname{var}(\hat{\alpha}) & \operatorname{cov}(\hat{\alpha}, \hat{\beta}) & \operatorname{cov}(\hat{\alpha}, \hat{\lambda}) \\
\operatorname{cov}(\hat{\alpha}, \hat{\beta}) & \operatorname{var}(\hat{\beta}) & \operatorname{cov}(\hat{\beta}, \hat{\lambda}) \\
\operatorname{cov}(\hat{\alpha}, \hat{\lambda}) & \operatorname{cov}(\hat{\beta}, \hat{\lambda}) & \operatorname{var}(\hat{\lambda})
\end{array}\right)
$$

Hence from the asymptotic normality of MLEs, approximate 100(1- $\alpha) \%$ confidence intervals for $\alpha, \beta$ and $\lambda$ can be constructed as,

$$
\hat{\alpha} \pm Z_{\alpha / 2} S E(\hat{\alpha}), \hat{\beta} \pm Z_{\alpha / 2} S E(\hat{\beta}) \text { and, } \hat{\lambda} \pm Z_{\alpha / 2} S E(\hat{\lambda})
$$

where $Z_{\alpha / 2}$ is the upper percentile of standard normal variate

\subsection{Method of Least-Square Estimation (LSE)}

For model parameters estimation of Beta distributions, the ordinary least square estimators and weighted least square estimators are proposed by Swain et al.(1988)

Here same procedure has been applied for the LIW distribution. The least-square estimators of the unknown parameters $\alpha, \beta$, and $\lambda$ of LIW distribution can be obtained by minimizing

$$
I(X ; \alpha, \beta, \lambda)=\sum_{i=1}^{n}\left[G\left(X_{i}\right)-\frac{i}{n+1}\right]^{2}
$$

with respect to unknown parameters $\alpha, \beta$, and $\lambda$. 
Let $G\left(X_{i}\right)$ denote the distribution function of the ordered random variables such that $\mathrm{X}_{(1)}<\mathrm{X}_{(2)}<\ldots<\mathrm{X}_{(\mathrm{n})}$ where $\left\{X_{1}, X_{2}, \ldots, X_{n}\right\}$ is a random sample of size $\mathrm{n}$ generated from a distribution function $\mathrm{G}($.). Then the least-square estimators of $\alpha, \beta$, and $\lambda$ say $\hat{\alpha}, \hat{\beta}$ and $\hat{\lambda}$ respectively, can be obtained by minimizing

$I(X ; \alpha, \beta, \lambda)=\sum_{i=1}^{n}\left[\frac{1}{1+\left(e^{\lambda x_{i}^{-\beta}}-1\right)^{\alpha}}-\frac{i}{n+1}\right]^{2} ; x \geq 0,(\alpha, \beta, \lambda>0)$.

with respect to $\alpha, \beta$, and $\lambda$.

Differentiating (3.2.2) with respect to $\alpha, \beta$, and $\lambda$ we get,

$$
\begin{aligned}
& \frac{\partial I}{\partial \alpha}=-2 \sum_{i=1}^{n}\left[\frac{1}{1+\left(e^{\lambda x_{i}^{-\beta}}-1\right)^{\alpha}}-\frac{i}{n+1}\right] \frac{\left[e^{\lambda x_{i}^{-\beta}}-1\right]^{\alpha} \ln \left[e^{\lambda x_{i}^{-\beta}}-1\right]}{\left\{1+\left[e^{\lambda x_{i}^{-\beta}}-1\right]^{\alpha}\right\}^{2}} \\
& \frac{\partial I}{\partial \beta}=-2 \alpha \lambda \sum_{i=1}^{n} \ln \left(x_{i}\right)\left[\frac{1}{1+\left(e^{\lambda x_{i}^{-\beta}}-1\right)^{\alpha}}-\frac{i}{n+1}\right] \frac{\left[e^{\lambda x_{i}^{-\beta}}-1\right]^{\alpha-1} e^{\lambda x_{i}^{-\beta}} x_{i}^{-\beta}}{\left\{1+\left[e^{\lambda x_{i}^{-\beta}}-1\right]^{\alpha}\right\}^{2}} \\
& \frac{\partial I}{\partial \lambda}=-2 \alpha \sum_{i=1}^{n}\left[\frac{1}{1+\left(e^{\lambda x_{i}^{-\beta}}-1\right)^{\alpha}}-\frac{i}{n+1}\right]\left[\frac{\left.e^{\lambda x_{i}^{-\beta}}-1\right]^{\alpha-1} e^{\lambda x_{i}^{-\beta}} x_{i}^{-\beta}}{\left\{1+\left[e^{\lambda x_{i}^{-\beta}}-1\right]^{\alpha}\right\}^{2}}\right.
\end{aligned}
$$

Similarly, the weighted least square estimators can be obtained by minimizing

$$
I(X ; \alpha, \beta, \lambda)=\sum_{i=1}^{n} w_{i}\left[G\left(X_{(i)}\right)-\frac{i}{n+1}\right]^{2}
$$

with respect to $\alpha, \beta$, and $\lambda$. The weights $w_{i}$ are $w_{i}=\frac{1}{\operatorname{Var}\left(X_{(i)}\right)}=\frac{(n+1)^{2}(n+2)}{i(n-i+1)}$

Hence, the weighted least square estimators of $\alpha, \beta$, and $\lambda$ respectively can be obtained by minimizing,

$$
I(X ; \alpha, \beta, \lambda)=\sum_{i=1}^{n} \frac{(n+1)^{2}(n+2)}{i(n-i+1)}\left[\frac{1}{1+\left(e^{\lambda x_{i}^{-\beta}}-1\right)^{\alpha}}-\frac{i}{n+1}\right]^{2}
$$

with respect to $\alpha, \beta$, and $\lambda$. 


\subsection{Method of Cramer-Von-Mises estimation (CVME)}

The CVME estimators of $\alpha, \beta$, and $\lambda$ of LIW distribution are obtained by minimizing the function

$$
\begin{aligned}
& M(X ; \alpha, \beta, \lambda)=\frac{1}{12 n}+\sum_{i=1}^{n}\left[G\left(x_{i: n} \mid \alpha, \beta, \lambda\right)-\frac{2 i-1}{2 n}\right]^{2} ; x \geq 0,(\alpha, \beta, \lambda>0) \\
& =\frac{1}{12 n}+\sum_{i=1}^{n}\left[\frac{1}{1+\left(e^{\lambda x_{i}^{-\beta}}-1\right)^{\alpha}}-\frac{2 i-1}{2 n}\right]^{2}
\end{aligned}
$$

Differentiating (3.3.1) with respect to $\alpha, \beta$, and $\lambda$ we get,

$$
\begin{aligned}
& \frac{\partial M}{\partial \alpha}=-2 \sum_{i=1}^{n}\left[\frac{1}{1+\left(e^{\lambda x_{i}^{-\beta}}-1\right)^{\alpha}}-\frac{2 i-1}{2 n}\right] \frac{\left[e^{\lambda x_{i}^{-\beta}}-1\right]^{\alpha} \ln \left[e^{\lambda x_{i}^{-\beta}}-1\right]}{\left\{1+\left[e^{\lambda x_{i}^{-\beta}}-1\right]^{\alpha}\right\}^{2}} \\
& \frac{\partial M}{\partial \beta}=-2 \alpha \lambda \sum_{i=1}^{n} \ln \left(x_{i}\right)\left[\frac{1}{1+\left(e^{\lambda x_{i}^{-\beta}}-1\right)^{\alpha}}-\frac{2 i-1}{2 n}\right] \frac{\left[e^{\lambda x_{i}^{-\beta}}-1\right]^{\alpha-1} e^{\lambda x_{i}^{-\beta}} x_{i}^{-\beta}}{\left\{1+\left[e^{\lambda x_{i}^{-\beta}}-1\right]^{\alpha}\right\}^{2}} \\
& \frac{\partial M}{\partial \lambda}=-2 \alpha \sum_{i=1}^{n}\left[\frac{1}{1+\left(e^{\lambda x_{i}^{-\beta}}-1\right)^{\alpha}}-\frac{2 i-1}{2 n}\right] \frac{\left[e^{\lambda x_{i}^{-\beta}}-1\right]^{\alpha-1} e^{\lambda x_{i}^{-\beta}} x_{i}^{-\beta}}{\left\{1+\left[e^{\lambda x_{i}^{-\beta}}-1\right]^{\alpha}\right\}^{2}}
\end{aligned}
$$

Solving $\frac{\partial W}{\partial \alpha}=0, \frac{\partial W}{\partial \beta}=0$ and $\frac{\partial W}{\partial \lambda}=0$ simultaneously we will get the CVM estimators.

\section{ILLUSTRATION WITH A REAL DATASET}

Birnbaum and Saunders (1969) originally analyzed the data given below which represents the fatigue life of 6061-T6 aluminum coupons cut parallel to the direction of rolling and oscillated at 18 cycles per second (cps) which consists of 101 observations with maximum stress per cycle 31,000 psi.

70, 90, 96, 97, 99, 100, 103, 104, 104, 105, 107, 108, 108, 108, 109, 109, 112, $112,113,114,114,114,116,119,120,120,120,121,121,123,124,124,124,124$, $124,128,128,129,129,130,130,130,131,131,131,131,131,132,132,132,133$, $134,134,134,134,134,136,136,137,138,138,138,139,139,141,141,142,142$, $142,142,142,142,144,144,145,146,148,148,149,151,151,152,155,156,157$, 157, 157, 157, 158, 159, 162, 163, 163, 164, 166, 166, 168, 170, 174, 196, 212. 
The MLEs are calculated directly by using the optim() function in R software (R Core Team, 2020) and (Ming, 2019) by maximizing the likelihood function (3.1). By maximizing the likelihood function in (3.1) we have obtained $\hat{\alpha}=22.2025, \hat{\beta}=$ $0.3451, \hat{\lambda}=3.7422$ and corresponding Log-Likelihood value is $l=-455.9623$. In Table 1 we have demonstrated the MLE's, standard errors (SE), and 95\% confidence interval for $\alpha, \beta$, and $\lambda$.

Table 1: MLE and SE and 95\% confidence interval for $\alpha, \beta$, and $\lambda$

\begin{tabular}{lll}
\hline \multicolumn{2}{l}{ Parameter MLE } & \multicolumn{1}{l}{ SE $95 \%$ ACI } \\
\hline alpha & 22.2025 & $4.47948(13.4227,30.9822)$ \\
beta & 0.3451 & $0.07461(0.1988,0.4913)$ \\
lambda & 3.7422 & $1.36437(1.0680,6.4163)$ \\
\hline
\end{tabular}

We have shown the graph of the profile log-likelihood function of $\alpha, \beta$, and $\lambda$ in Figure 2 and observed that the MLEs are unique.
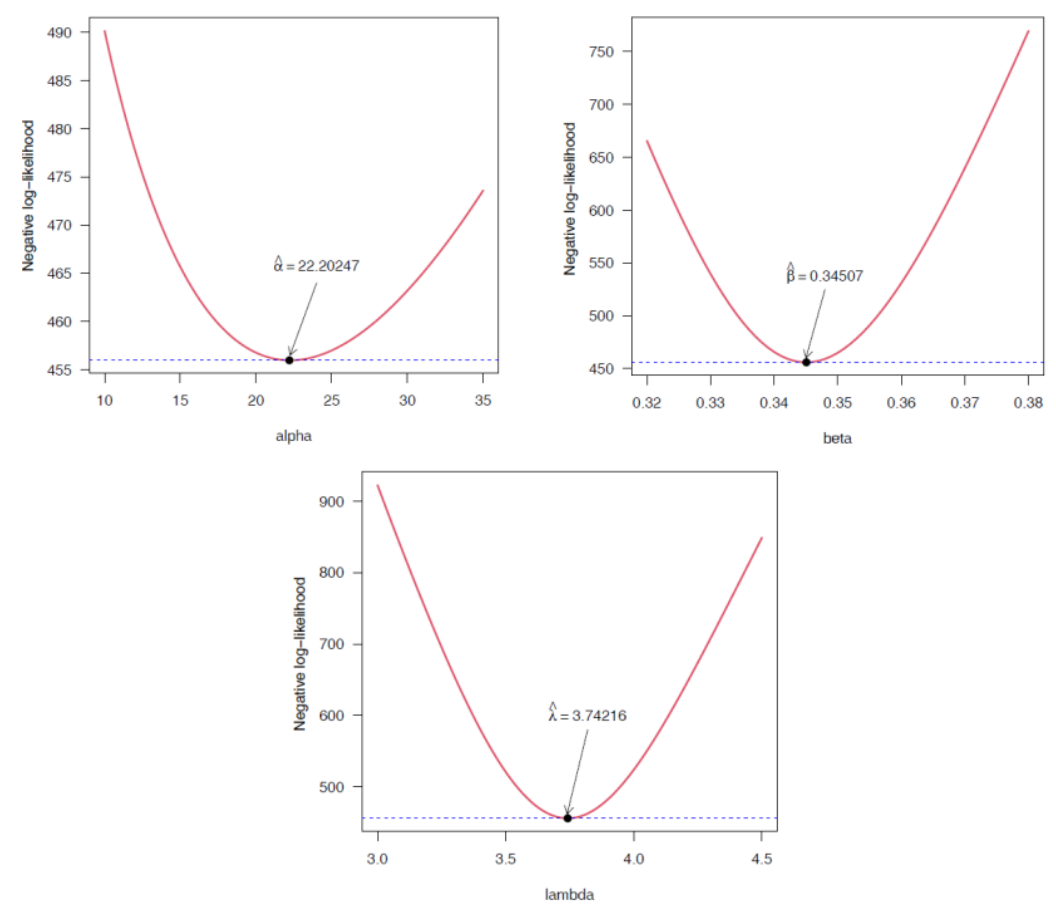

Figure 2. Graph of profile log-likelihood function of $\alpha, \beta$, and $\lambda$. 
In Figure 3 we have presented the Q-Q plot (empirical quantile against theoretical quantile) and CDF plot (empirical distribution function against theoretical distribution function).
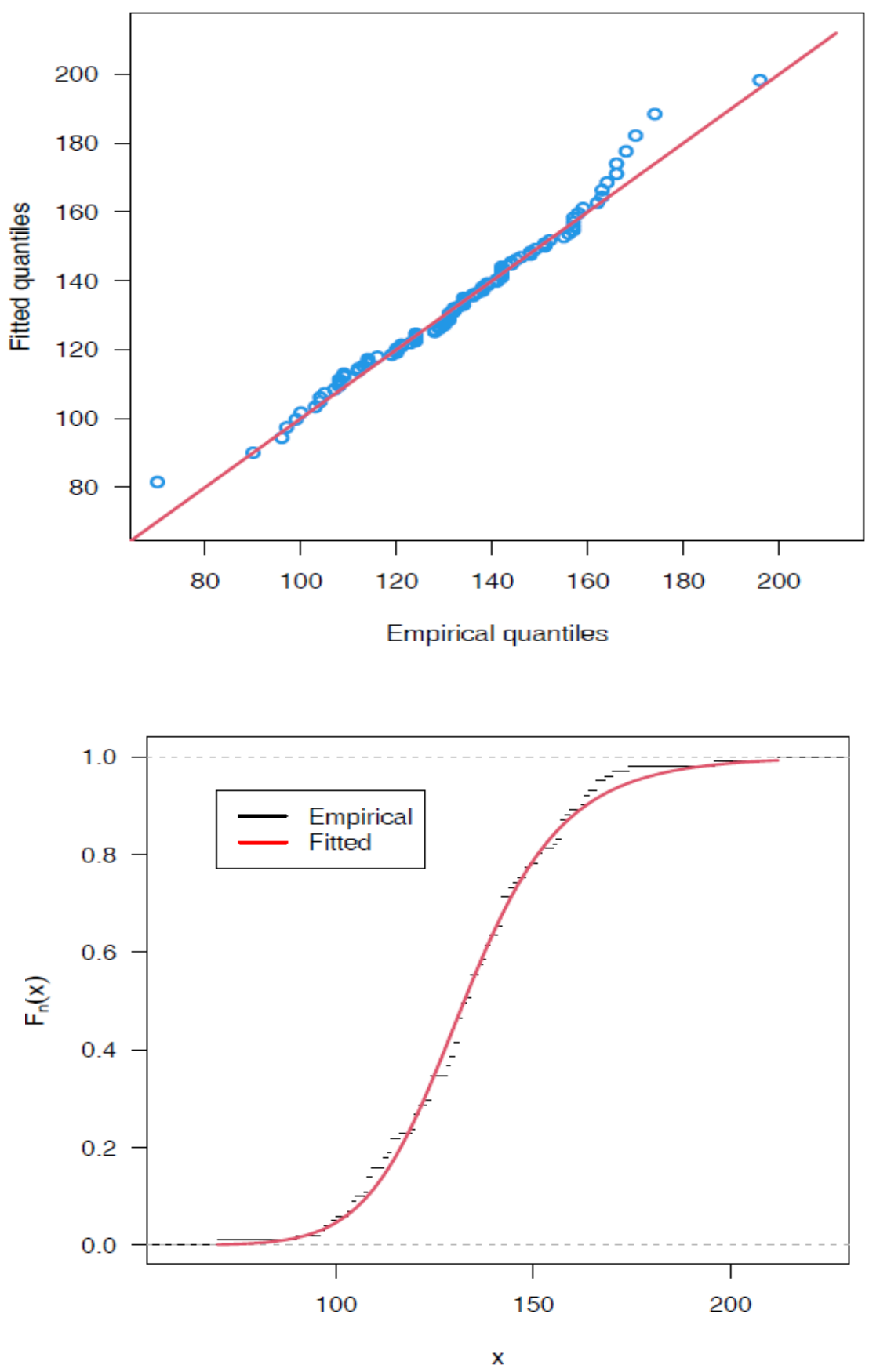

Figure 3. The Q-Q plot (upper panel) and CDF plot (lower panel) of LEE distribution 
By using MLE, LSE, and CVME methods we estimate the parameter of LIW distribution. For the goodness of fit purpose, we use negative log-likelihood (-LL), Bayesian information criterion (BIC), Akaike information criterion (AIC), HannanQuinn information criterion (HQIC), and Corrected Akaike Information Criterion (AICC) statistic to select the best model among selected models. The expressions to calculate AIC, BIC, AICC, and HQIC are listed below.
a) $A I C=-2 l(\hat{\theta})+2 k$
b) $B I C=-2 l(\hat{\theta})+k \log (n)$
c) $A I C C=A I C+\frac{2 k(k+1)}{n-k-1}$
d) $H Q I C=-2 l(\hat{\theta})+2 k \log [\log (n)]$

where $\mathrm{k}$ denotes the number of parameters and $\mathrm{n}$ denotes the size of the sample in the model under consideration. Further, to evaluate the fits of the LIW distribution with some selected distributions we have taken the Anderson-Darling (W), KolmogorovSimnorov (KS), and the Cramer-Von Mises $\left(\mathrm{A}^{2}\right)$ statistic.

Non-nested models are compared usually using these statistics values and these statistics also help to demonstrate how closely a specific CDF fits the empirical distribution of a given real data set. These statistics are calculated as

$$
\begin{aligned}
& K S=\max _{1 \leq i \leq n}\left(d_{i}-\frac{i-1}{n}, \frac{i}{n}-d_{i}\right) \\
& W=-n-\frac{1}{n} \sum_{i=1}^{n}(2 i-1)\left[\ln d_{i}+\ln \left(1-d_{n+1-i}\right)\right] \\
& A^{2}=\frac{1}{12 n}+\sum_{i=1}^{n}\left[\frac{(2 i-1)}{2 n}-d_{i}\right]^{2}
\end{aligned}
$$

where $d_{\mathrm{i}}=C D F\left(x_{\mathrm{i}}\right)$; the $\mathrm{x}_{\mathrm{i}}$ 's being the ordered observations.

In Table 2 we have displayed the estimated value of the parameters of Logistic inverse exponential distribution using MLE, LSE, and CVME method and their corresponding negative log-likelihood, AIC, BIC, AICC, and HQIC information criteria. 
A Study on Properties and Goodness-of-Fit of the Logistic Inverse...

Table 2: Estimated parameters, log-likelihood, AIC, BIC, AICC and HQIC

\begin{tabular}{lllllllll}
\hline $\begin{array}{l}\text { Method of } \\
\text { Estimation }\end{array}$ & $\hat{\alpha}$ & $\hat{\beta}$ & $\hat{\lambda}$ & -LL & AIC & BIC & AICC & IC \\
\hline MLE & 22.2025 & 0.3451 & 3.7422 & 455.9623 & 917.9246 & 925.7699 & 918.172 & 921.1006 \\
LSE & 16.9627 & 0.4436 & 6.0636 & 456.0685 & 918.1371 & 925.9824 & 918.3845 & 921.3131 \\
CVME & 16.5590 & 0.4623 & 6.6427 & 456.0548 & 918.1095 & 925.9549 & 918.3569 & 921.2855 \\
\hline
\end{tabular}
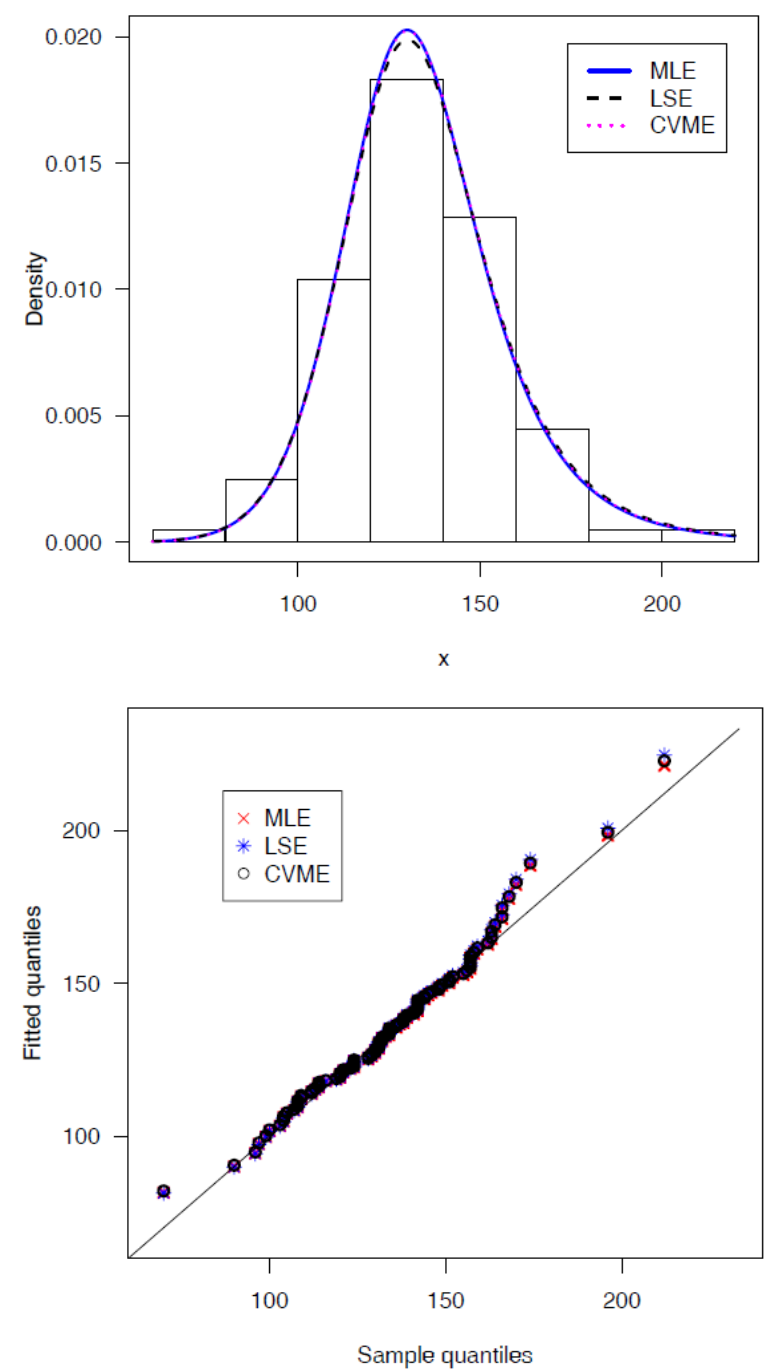

Figure 4. The Histogram and the density function of fitted distributions (upper panel) and Q-Q plot of estimation methods MLE, LSE, and CVME (lower panel). 
Table 3. The KS, AD and CVM statistic with p-value

\begin{tabular}{llll}
\hline $\begin{array}{l}\text { Method of } \\
\text { Estimation }\end{array}$ & KS $(\boldsymbol{p}$-value $)$ & $\boldsymbol{A D}(\boldsymbol{p}$-value $)$ & $\boldsymbol{C V M}(\boldsymbol{p}$-value $)$ \\
\hline MLE & $0.0637(0.8072)$ & $0.0532(0.8576)$ & $0.3735(0.8743)$ \\
LSE & $0.0583(0.8821)$ & $0.0522(0.8641)$ & $0.3939(0.8544)$ \\
CVME & $0.0577(0.8901)$ & $0.0517(0.8672)$ & $0.3947(0.8536)$ \\
\hline
\end{tabular}

To illustrate the goodness of fit of the Lindley inverse Weibull distribution, we have taken some well-known distribution for comparison purpose which is listed blew,

\section{Generalized Rayleigh distribution}

The PDF of Generalized Rayleigh (GR) distribution (Kundu \& Raqab, 2005) is $f_{G R}(\mathrm{x} ; \alpha, \lambda)=2 \alpha \lambda^{2} \mathrm{x}^{-(\lambda x)^{2}}\left\{1-\mathrm{e}^{-(\lambda x)^{2}}\right\}^{\alpha-1} ;(\alpha, \lambda)>0, x>0$

Here the respective scale and shape parameters are denoted by $\lambda$ and $\alpha$.

\section{Chen distribution}

The PDF of Chen distribution is presented by (Chen, 2000) as

$$
f_{C N}(x ; \lambda, \beta)=\lambda \beta x^{\beta-1} e^{x^{\beta}} \exp \left\{\lambda\left(1-e^{x^{\beta}}\right)\right\} ;(\lambda, \beta)>0, x>0 \text {. }
$$

\section{Generalized Exponential (GE) distribution}

The PDF of generalized exponential distribution (Gupta \& Kundu, 1999)

$$
f_{G E}(x ; \alpha, \lambda)=\alpha \lambda e^{-\lambda x}\left\{1-e^{-\lambda x}\right\}^{\alpha-1} ;(\alpha, \lambda)>0, x>0 .
$$

\section{Exponential power (EP) distribution}

The PDF Exponential power (EP) distribution (Smith \& Bain, 1975) is

$$
f_{E P}(x)=\alpha \lambda^{\alpha} x^{\alpha-1} e^{(\lambda x)^{\alpha}} \exp \left\{1-e^{(\lambda x)^{\alpha}}\right\} ;(\alpha, \lambda)>0, \quad x \geq 0 .
$$

Here the respective scale and shape parameters are denoted by $\lambda$ and $\alpha$. For the assessment of the potentiality of the proposed model, we have presented the value of the AIC, BIC, AICC, and HQIC which are presented in Table 4. 
Table 4. Log-likelihood (LL), AIC, BIC, AICC and HQIC

\begin{tabular}{clcccc}
\hline Model & -LL & AIC & BIC & AICC & HQIC \\
\hline LIW & 455.9623 & 917.9246 & 925.7699 & 918.1720 & 921.1006 \\
GR & 457.3766 & 918.7532 & 923.9835 & 918.8757 & 920.8706 \\
GE & 463.7324 & 931.4648 & 936.6951 & 931.5873 & 933.5822 \\
Chen & 467.0598 & 938.1196 & 943.3499 & 938.2421 & 940.2370 \\
EP & 476.7897 & 957.5794 & 962.8096 & 957.6994 & 959.6967 \\
\hline
\end{tabular}

The Histogram and the density function of fitted distributions and Empirical distribution function with the estimated distribution function of LIW and some selected distributions are presented in Figure 6.
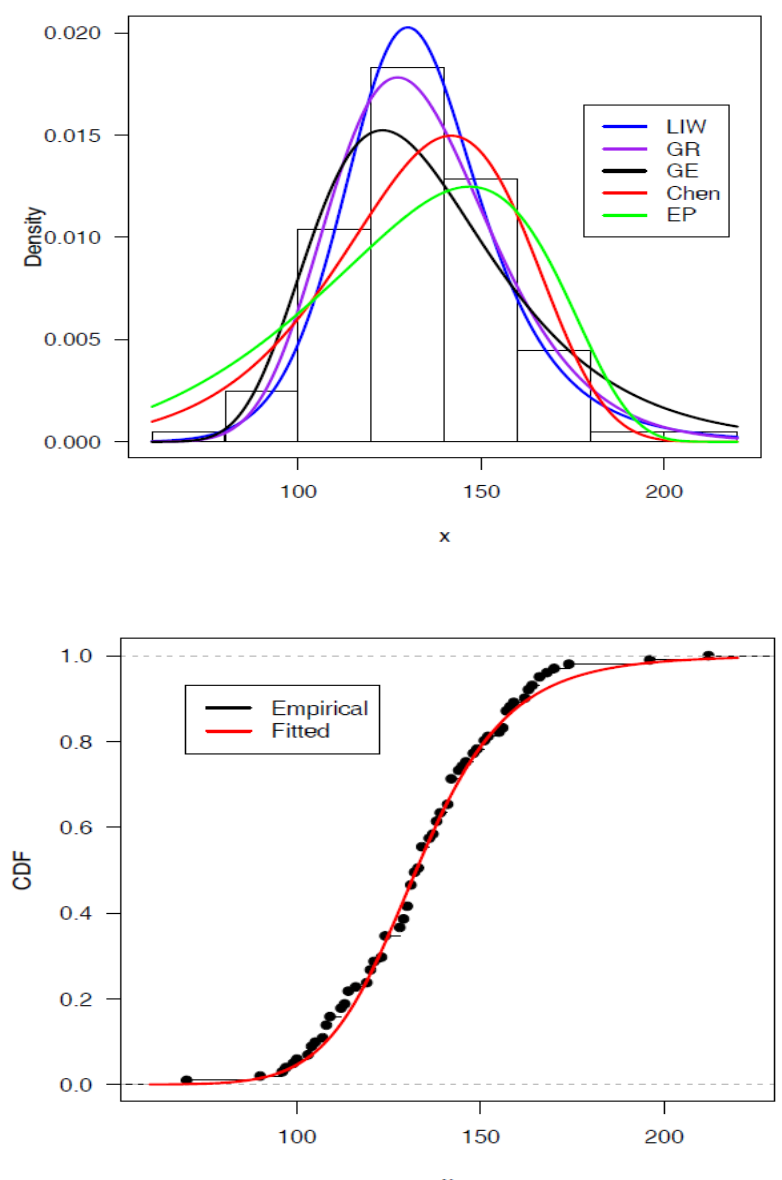

Figure 6. The Histogram and the density function of fitted distributions (upper panel) and Empirical distribution function with estimated distribution function (lower panel). 
In Table 5, we have presented the value of Kolmogorov-Simnorov (KS), the CramerVon Mises (CVM), and the Anderson-Darling (AD) statistics to compare the goodness-of-fit of the LIW distribution with other well-known existing distributions. It is observed that the LIW distribution has the minimum value of the test statistic and higher $p$-value thus we conclude that the LIW distribution gets quite better fit and more consistent and reliable results from others taken for comparison.

Table 5. The goodness-of-fit statistics and their corresponding p-value

\begin{tabular}{clll}
\hline Model & KS $(\boldsymbol{p}$-value $)$ & $\boldsymbol{C V M}(\boldsymbol{p}$-value $)$ & $\boldsymbol{A D}(\boldsymbol{p}$-value $)$ \\
\hline LIW & $0.0637(0.8072)$ & $0.3735(0.8743)$ & $0.0532(0.8576)$ \\
GR & $0.0901(0.3850)$ & $0.6033(0.6445)$ & $0.1050(0.5620)$ \\
GE & $0.1066(0.2014)$ & $2.0724(0.0840)$ & $0.3112(0.1257)$ \\
Chen & $0.1102(0.1718)$ & $2.0769(0.0835)$ & $0.2960(0.1386)$ \\
EP & $0.1378(0.0433)$ & $4.5057(0.0050)$ & $0.6942(0.0130)$ \\
\hline
\end{tabular}

\section{CONCLUSIONS}

In this research work, we have introduced a three-parameter univariate continuous distribution termed Logistic Inverse Weibull distribution. We have presented some statistical properties of the distribution such as the cumulative distribution function, probability density function and survival function, hazard rate function, quantile function, skewness, and kurtosis measures. We have employed the three well-known estimation methods namely MLE, CVME, and LSE methods for the model parameter estimation and we concluded that the MLEs are quite better than LSE, and CVM. A real data set is taken to investigate the application and suitability of the proposed distribution and found that the proposed model has quite better goodness of fit than other lifetime model taken into consideration. We hope this model may be an alternative in the field of survival analysis, probability theory, and applied statistics.

\section{REFERENCES}

[1] Abd EL-Baset, A. A., \& Ghazal, M. G. M. (2020). Exponentiated additive Weibull distribution. Reliability Engineering \& System Safety, 193, 106663.

[2] Afify, A. Z., Nofal, Z. M., \& Butt, N. S. (2014). Transmuted complementary Weibull geometric distribution. Pakistan Journal of Statistics and Operation Research, 435-454. 
[3] Akgül, F. G., Şenoğlu, B., \& Arslan, T. (2016). An alternative distribution to Weibull for modeling the wind speed data: Inverse Weibull distribution. Energy Conversion and Management, 114, 234-240.

[4] Aryal, G. R., Ortega, E. M., Hamedani, G. G., \& Yousof, H. M. (2017). The Topp-Leone generated Weibull distribution: regression model, characterizations, and applications. International Journal of Statistics and Probability, 6(1), 126-141.

[5] Aryal, G. R., \& Tsokos, C. P. (2011). Transmuted Weibull distribution: A generalization of the Weibull probability distribution. European Journal of Pure and Applied Mathematics, 4(2), 89-102.

[6] Basheer, A. M. (2019). Alpha power inverse Weibull distribution with reliability application. Journal of Taibah University for Science, 13(1), 423432.

[7] Birnbaum, Z.W., \& Saunders, S.C. (1969). Estimation for a family of life distributions with applications to fatigue, Journal of Applied Probability, 6, $328-347$.

[8] Casella, G., \& Berger, R. L. (1990). Statistical Inference. Brooks/ Cole Publishing Company, California.

[9] Chen, Z. (2000). A new two-parameter lifetime distribution with bathtub shape or increasing failure rate function, Statistics \& Probability Letters, 49, $155-161$.

[10] Cordeiro, G. M., Ortega, E. M., \& da Cunha, D. C. (2013). The exponentiated generalized class of distributions. Journal of Data Science, 11(1), 1-27.

[11] Cordeiro, G. M., Afify, A. Z., Yousof, H. M., Cakmakyapan, S., \& Ozel, G. (2018). The Lindley Weibull distribution: properties and applications. Anais da Academia Brasileira de Ciências, 90(3), 2579-2598.

[12] Ghitany, M. E., Al-Hussaini, E. K., \& Al-Jarallah, R. A. (2005). MarshallOlkin extended Weibull distribution and its application to censored data. Journal of Applied Statistics, 32(10), 1025-1034.

[13] Glantz, M. \& Kissell, R. (2014). A Primer on Risk Mathematics. In M. Glantz \& R. Kissell(Eds.), Multi-Asset Risk Modeling (pp.21-61).Elsevier Inc.

[14] Gupta, R. D., and Kundu, D. (1999). Generalized exponential distributions, Australian and New Zealand Journal of Statistics, 41(2), 173 - 188.

[15] Hanook, S., Shahbaz, M. Q., Mohsin, M., \& Golam Kibria, B. M. (2013). A note on beta inverse-Weibull distribution. Communications in StatisticsTheory and Methods, 42(2), 320-335.

[16] Khan, M. S., Pasha, G. R., \& Pasha, A. H. (2008). Theoretical analysis of inverse Weibull distribution. WSEAS Transactions on Mathematics, 7(2), 3038 . 
[17] Kumar, K., \& Kumar, I. (2019). Estimation in inverse Weibull distribution based on randomly censored data. Statistica, 79(1), 47-74.

[18] Kundu, D. \& Raqab, M. Z. (2005). Generalized Rayleigh distribution: different methods of estimations. Comp. Stat. \& Data Anal., 49, 187 - 200.

[19] Lai, C. D., Xie, M., \& Murthy, D. N. P. (2003). A modified Weibull distribution. IEEE Transactions on reliability, 52(1), 33-37.

[20] Lan, Y., \& Leemis, L. M. (2008). The logistic-exponential survival distribution. Naval Research Logistics (NRL), 55(3), 252-264.

[21] Lee, C., Famoye, F., \& Olumolade, O. (2007). Beta-Weibull distribution: some properties and applications to censored data. Journal of modern applied statistical methods, 6(1), 17

[22] Ming Hui, E. G. (2019). Learn R for applied statistics. Springer, New York.

[23] Mudholkar, G. S., Srivastava, D. K., \& Kollia, G. D. (1996). A generalization of the Weibull distribution with application to the analysis of survival data. Journal of the American Statistical Association, 91(436), 1575-1583.

[24] Nadarajah, S. (2009). Bathtub-shaped failure rate functions. Qual Quant 43: 855-863.

[25] Okasha, H. M., El-Baz, A. H., Tarabia, A. M. K., \& Basheer, A. M. (2017). Extended inverse Weibull distribution with reliability application. Journal of the Egyptian Mathematical Society, 25(3), 343-349.

[26] Pararai, M., Warahena-Liyanage, G., \& Oluyede, B. O. (2014). A new class of generalized inverse Weibull distribution with applications. Journal of Applied Mathematics and Bioinformatics, 4(2), 17.

[27] R Core Team (2020). R: A language and environment for statistical computing. R Foundation for Statistical Computing, Vienna, Austria. URL https://www.R-project.org/.

[28] Sarhan, A. M., \& Zaindin, M. (2009). Modified Weibull distribution. APPS. Applied Sciences, 11, 123-136.

[29] Shahbaz, M. Q., Shahbaz, S., \& Butt, N. S. (2012). The KumaraswamyInverse Weibull Distribution. Shahbaz, MQ, Shahbaz, S., \& Butt, NS (2012). The Kumaraswamy-Inverse Weibull Distribution. Pakistan journal of statistics and operation research, 8(3), 479-489. repeat

[30] Silva, G. O., Ortega, E. M., \& Cordeiro, G. M. (2010). The beta modified Weibull distribution. Lifetime data analysis, 16(3), 409-430.

[31] Smith, R.M., and Bain, L.J. (1975). An exponential power life-test distribution, Communications in Statistics, 4, 469-481.

[32] Swain, J. J., Venkatraman, S. \& Wilson, J. R. (1988). Least-squares estimation of distribution functions in johnson's translation system. Journal of Statistical Computation and Simulation, 29(4), 271-297. 
[33] Tahir, M. H., Cordeiro, G. M., Alzaatreh, A., Mansoor, M., \& Zubair, M. (2016). The logistic-X family of distributions and its applications. Communications in Statistics-Theory and Methods, 45(24), 73267349.

[34] Xie, M., \& Lai, C. D. (1996). Reliability analysis using an additive Weibull model with a bathtub-shaped failure rate function. Reliability Engineering \& System Safety, 52(1), 87-93. 
